\title{
NUMERICAL SIMULATION AND INVESTIGATION OF MIXING DURING RELEASE OF DENSE FLUID INTO AMBIENT FLUID
}

\author{
R. R. C. SANTOS, S. S. V. VIANNA \\ University of Campinas, School of Chemical Engineering \\ Email para contato: savio@feq.unicamp.br \\ rap11@feq.unicamp.br
}

\begin{abstract}
Numerical simulations using non-commercial OpenFOAM software were performed and compared with experimental data. The experiment consisted in pumping dense fluid (saline solution of sodium chloride and food dye) into a tank with a less dense fluid (fresh water) next to the bottom of the tank. The released fluid dilutes similarly to real cases of gas dispersion governed by buoyancy forces. Reynolds Averaged Navier-Stokes (RANS) approach with $k-\omega S S T$ model was applied to simulate three experimental cases with different initial conditions. The results were compared qualitatively and numerically concerning the height $\left(h_{t}\right)$ of the dense layer and its velocity profile. The numerical findings agree with the experimental data.
\end{abstract}

\section{INTRODUCTION}

The release of fluid from a region with higher pressure to a lower pressure region leads to the hydraulic jump. This concept is most commonly used concerning nonmiscible fluids, for example, water flowing along a channel with air (Gualtieri and Chanson (2007)), ie., a gas-liquid system. Similar effect happens with miscible fluids, however the mixing process leads to the dilution of the dense released fluid. In case of flammable gases releases, the dilution of the released fluid next to the source poses an additional issue to accidental releases, as the fluid would reach the upper flammability limit quicker and closer to the release point.

The dilution rate depends on the dense fluid release conditions and properties. In the case of fluids with density difference - flow governed by buoyancy - the Froude number $(F r)$ can be related with the dilution rate or ambient fluid entertainment rate due to the mixing process (Sher and Woods (2017)). Quantifying the dilution rate during the gas release as a function of $\mathrm{Fr}$ should help to improve models used to estimate the volume of heavy flammable gas clouds for engineering applications. Therefore, this work aims to simulate the release of dense fluid to obtain information concerning the dilution rate at the near field, ie., the region close to the dense fluid release source, as function of the initial Froude number $\operatorname{Fr} r_{0}=\operatorname{Fr}\left(x_{0}\right)$.

\section{METHODOLOGY}

Three experiments have been simulated. The experimental conditions are presented in Table 1. The experiments were selected regarding the initial Froude number $F r_{0}$, that present $F r_{0}>1(\mathrm{~A}), F r_{0} \approx 1$ (B) and $F r_{0}<1(\mathrm{C})$. The dense fluid 

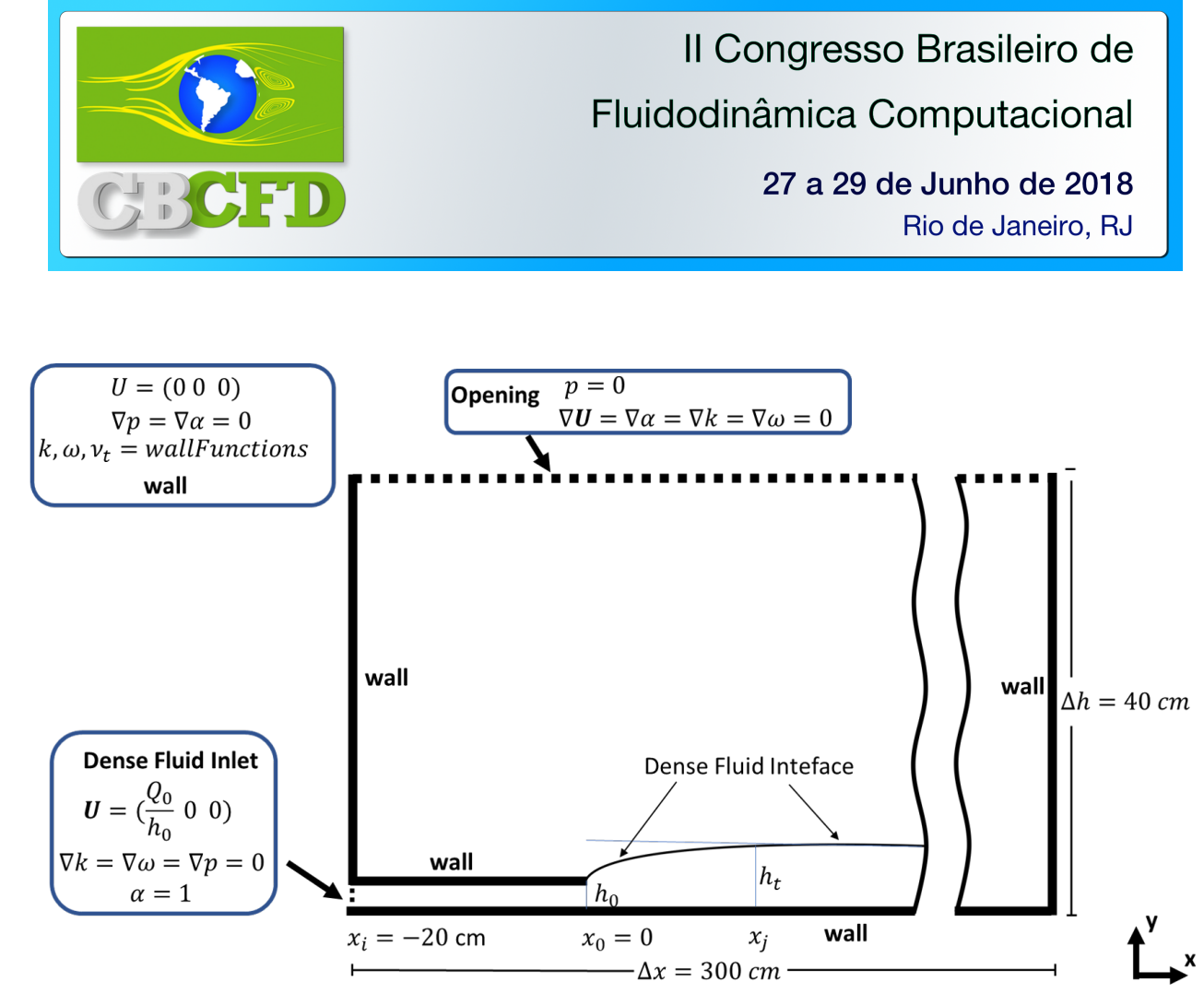

Figure 1 - Geometry and boundary conditions used to simulate the experimental cases. In the image is included the hydraulic jump effect for a case with $F r_{0}>1$ and the definitions of $x_{j}$ and $h_{t}$. $\mathbf{U}$ is the vector velocity, $p$ is the dynamic pressure, alpha means the fraction of dense fluid and $k, \omega, \nu_{t}$ are the turbulent parameters.

(sodium chloride saline solution with a well know concentration) is pumped into the tank with less dense fluid (fresh water). Food red dye is added to visualise the dense fluid. The square tube leading the fluid into the tank has height $h_{0}=2.8 \mathrm{~cm}$ and was positioned near the bottom of the tank. In this way, the dense fluid flows over the surface and a layer of stratified flow is formed. The height of the formed layer was measured since it indicates the dense fluid dilution rate.

Table 1 - Initial conditions of the experiments A, B and C. These conditions were used to perform the numerical simulations. The table shows the experiment name, the volume flux per unit of tank width, reduced gravity, Reynolds and Froude dimensionless numbers.

\begin{tabular}{|lllll|}
\hline Exp & $Q_{0}\left(\mathrm{~cm}^{2} . \mathrm{s}^{-1}\right)$ & $g_{0}^{\prime}\left(\mathrm{cm} . \mathrm{s}^{-1}\right)$ & $R e_{0}$ & $F r_{0}$ \\
\hline A & 29.5 & 8,65 & 2400 & 2.04 \\
B & 36.5 & 63,88 & 3600 & 0.93 \\
C & 29.5 & 78,92 & 2400 & 0.68 \\
\hline
\end{tabular}

The simulations were performed in two dimensions (2D) using the OpenFOAM v.4.1, specifically the twoLiquidMixingFoam solver, which was chosen because present an incompressible multi-species model for miscible fluids. In this case, molecular diffusivity used was $D_{A B}=10^{-9} \mathrm{~m}^{2} / \mathrm{s}$. The turbulence was modelled using $k-\omega S S T$ - The geometry was developed as in the experimental case exhibited in Figure 1, which is divided in two regions, (i) an initial pipe region $\left.\Delta x\right|_{\left(x<x_{0}\right)}=20 \mathrm{~cm}$ and $h=h_{0}=2.8 \mathrm{~cm}$, and (ii) the open region $\left.\Delta x\right|_{\left(x>x_{0}\right)}=3000 \mathrm{~cm}$ and $h=14 h_{0}$. The mesh independence study was used to obtain the best computational cell dimensions. 
For all cases presented here, a structured mesh with cell dimensions $\delta x=\delta y=2 \mathrm{~mm}$ was used. The boundary conditions of dense fluid velocity and concentration were uniformly specified at the beginning of the pipe, however velocity and concentration profiles are already developed at $x_{0}$.

\section{RESULTS}

Numerical simulations were performed with the setup described in the previous section. The comparison between experimental data and numerical simulation was based on the height of the dense fluid layer $h_{t}=h\left(x_{j}\right)$. The comparison was made considering the steady state of both cases, experimental and simulation. The position $x_{j}$ was chosen based on experiment A, defined as $f\left(x_{j}=\min (x)\right)=0$ where, $f(x)=d h /\left.d x\right|_{x}$ of the experiment $\mathrm{A}$, representing the position on $x$-axis relative to the end of the effects on the height of the layer caused by the hydraulic jump formation. The same $x_{j}$ was set for all experiments in order to compare the three cases. For the simulations, $h_{t s} i m=h\left(x_{j}\right)$. Figure 2 shows the comparison between experiment and simulation, indicating the relevant variables to the simulation. The flow behaviour was well captured as the variation of the height as function of Froude number is properly observed in the analysis of the plots presented in Figure 2.

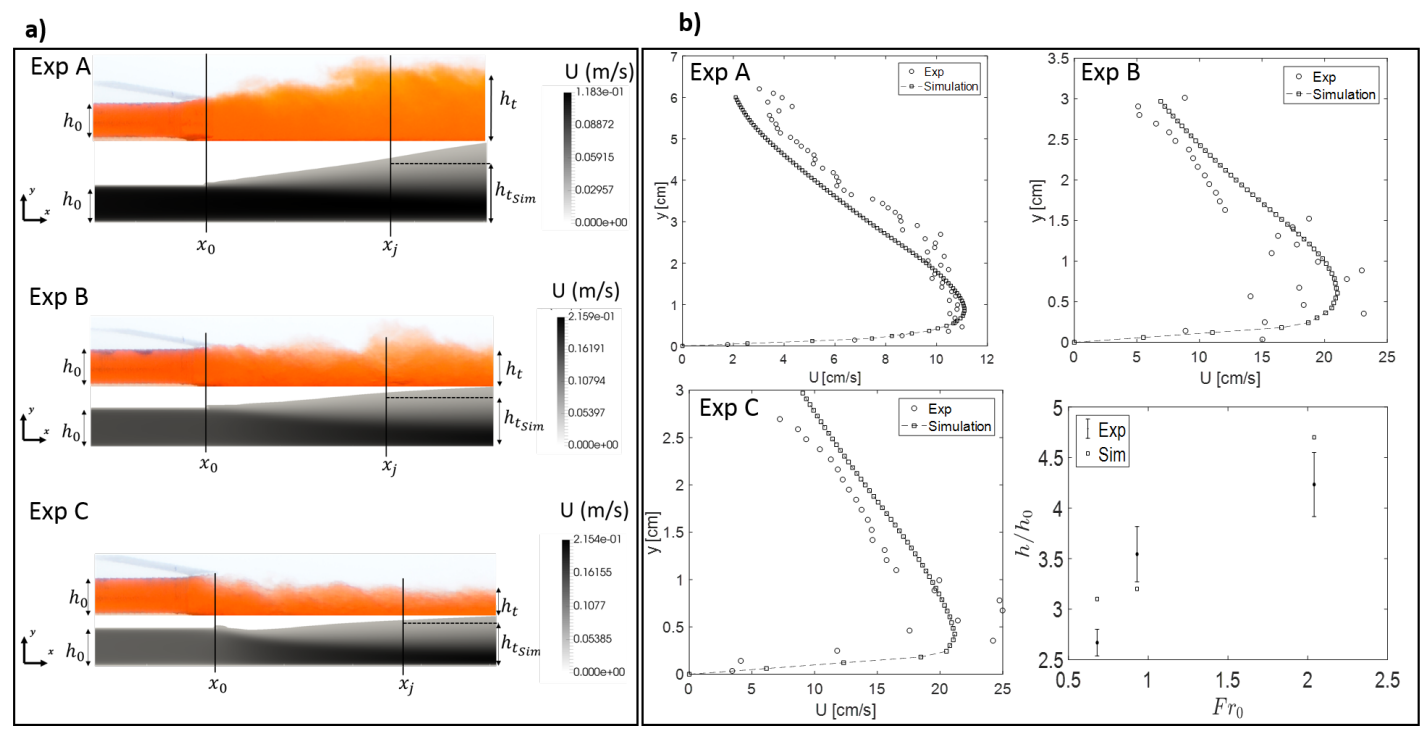

Figure 2 - Comparison between experiment and numerical simulation. The images are presented in a) for the three experiments and show dense fluid structure when the flow reaches steady state. The experimental images show the fluid released in red-orange colour, while images of the simulations presents the velocity in gray scale. b) Velocity profiles from experiments and simulation in the dense layer is plotted against its height. The last image shows the dimensionless height relative to the pipe height $h_{t} / h_{0}$ and $h_{t s i m} / h_{0}$ as a function of the initial Froude number. The relative error between experiment and simulation, using the relative heights as comparison parameter, for the experiments $\mathrm{A}, \mathrm{B}$ and $\mathrm{C}$ are $11 \%, 10 \%$ and $16 \%$, respectively 
The images of the simulations show the velocity field distribution within the flow region, showing that the experiments with higher density tend to increase velocity near the wall after the release. The comparison between the velocity profiles and heights of the dense fluid layer are plotted in Figure $2 \mathrm{~b}$ ), which it is noticed that both experiment and simulation show the same trend as function of the Froude number. The larger the Froude number, the greater the height of the layer. The same flow behaviour applies to the opposite case, the lower the Froude number, the smaller the height, however the simulations seem to struggle to properly represent the flow for low Froude number.

\section{CLOSING REMARKS}

Numerical dense fluid release simulations were performed using the OpenFOAM twoLiquidMixingFoam solver. Although the experimental data concerning the velocity profiles carry a bit of noise in the reported measurements, the simulations presented good agreement with experimental data. The solver shows good results for predicting the height of the dense fluid layer, which indicates the mixing between the fluids. The simulations can be used to calculate the dilution rate and later a model can be developed to predict the volume of fluid released near source region.

\section{NOMECLATURE}

$Q$ - Volume flux per unit of width of the tank at $\mathrm{cm}^{2} / \mathrm{s}$.

$h$ - Height relative to the $y$-axis in $\mathrm{cm}$.

$x$ - Distance from the release point along the flow direction in $\mathrm{cm}$.

$F r$ - Froude number defined as $\left.F r=U / \sqrt{(} g^{\prime} h\right)$, where $g^{\prime}=g\left(\rho_{d}-\rho\right) / \rho$ is reduced gravity in $\mathrm{cm} / \mathrm{s}^{2} . g=982 \mathrm{~cm} / \mathrm{s}^{2}$ is the Earth gravity acceleration, $\rho$ is the density of the light fluid and $\rho_{d}$ is the density of the dense fluid.

$R e$ - Reynolds number defined as $R e=U h / \nu$, where $U$ is the flow velocity in $\mathrm{cm} / \mathrm{s}$ and $\nu=100 \mathrm{~cm}^{2} / \mathrm{s}$ is the kinematic viscosity.

\subsection{Subscripts}

0 - Regarding the initial condition, at the release source.

$j$ - Concerning the hydraulic jump.

$t$ - Distance from the release point along the flow direction in $\mathrm{cm}$.

\section{REFERENCES}

Gualtieri, C.; Chanson, H. Experimental analysis of Froude number effect on air entrainment in the hydraulic jump. Environmental Fluid Mechanics, 7(3), 217-238, 2007.

Sher, D.; Woods, A. W. Mixing in continuous gravity currents. Journal of Fluid Mechanics, 818(November 2016), R4, 2017. 